\title{
Reduced Length of Stay Following Patient Pathway Optimization for Primary Hip and Knee Arthroplasty at a Swiss Hospital
}

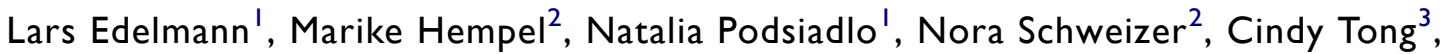 \\ Thibaut Galvain $\mathbb{D}^{4}$, Hannah Taylor $\mathbb{D}^{5}$, Michael Schüler ${ }^{1}$ \\ 'Kantonsspital Münsterlingen, Münsterlingen, 8596, Switzerland; ${ }^{2}$ Johnson \& Johnson AG, Zug, 6300, Switzerland; ${ }^{3}$ Johnson and Johnson Medical \\ Devices, Somerville, NJ, USA; ${ }^{4}$ Johnson and Johnson Medical, Issy les Moulineaux, France; ${ }^{5}$ Johnson \& Johnson Medical Limited, Berkshire, UK \\ Correspondence: Lars Edelmann, Klinik für Orthopädie und Traumatologie, Kantonsspital Münsterlingen, Spitalcampus I, Münsterlingen, 8596, \\ Switzerland, Tel +4I-7I-686-20 50, Fax +4I-7I-686-20 23, Email lars.edelmann@stgag.ch
}

Purpose: Total knee and hip arthroplasties (TKA and THA) are common surgical procedures in Switzerland and worldwide. Alongside increasing demand for these procedures, many providers have adopted enhanced recovery programs (ERPs) that aim to optimize recovery after these surgeries. We evaluated the impact of implementing a Patient Pathway Optimization (PPO) program designed around ERP principles for patients undergoing TKA or THA in a Swiss Cantonal hospital. The primary objective was to determine whether PPO implementation for patients undergoing TKA or THA reduced length of stay (LOS) during index hospitalization. Additional study outcomes were hospital inpatient costs, rate of complications and readmissions, and patient discharge destination.

Methods: This was a retrospective, observational study comparing outcomes before and after PPO implementation for patients who underwent primary TKA or THA from 2014 to 2019. The PPO program modified the care pathway and implemented five ERP principles (preoperative patient education, pre-operative physiotherapy, local infiltration analgesia, early mobilization, and oral analgesia). Hospital electronic medical record and billing data were used for the analysis.

Results: After implementation of PPO, LOS was shorter compared with the pre-PPO period (reduction of 2.5 and 2.4 days in TKA and THA cohorts, respectively, both $\mathrm{p}<0.001$ ). Mean inpatient costs per patient were also reduced following PPO (decrease of USD2016 [ $p=0.015]$ and USD340 [p $=0.54]$ for TKA and THA cohorts, respectively). There were no observed increases in readmissions or complications after PPO implementation in either the TKA or THA cohort. More patients in the post-PPO groups were discharged to home vs an alternative health-care setting than in the pre-PPO groups (TKA, 83.8\% vs 68.4\% [p $=0.07$ ]; THA, 80.4\% vs $73.9 \%,[\mathrm{p}=0.31])$.

Conclusion: Patient pathway optimization and implementation of additional enhanced recovery principles for TKA and THA may benefit both health systems and patients, by reducing LOS and costs without increases in complications or readmissions.

Keywords: enhanced recovery program, total hip replacement, total knee replacement, joint replacement, costs

\section{Introduction}

Total knee and total hip arthroplasty (TKA and THA) are effective treatments for relieving pain, restoring function and improving quality of life for patients with end-stage arthritis, and are among the most common surgical procedures performed worldwide. ${ }^{1}$ Switzerland has among the highest per-capita rates of these procedures in Europe, ${ }^{1}$ with 18,885 primary hip and 14,269 primary knee replacement operations performed in 2018. ${ }^{2}$ Demand for TKA and THA is growing $^{3-5}$ with THA procedure incidence in Switzerland projected to increase by $94 \%$ from 2015 to 2050 , due to broadening indications and aging populations. ${ }^{6}$ The incidence of TKA is predicted to increase by approximately $45 \%$ in other European countries over a similar time frame, also due to aging populations. ${ }^{7,8}$ 
Although widely regarded as clinically efficacious and cost-effective, ${ }^{9-11}$ the rising demand for TKA and THA procedures, coupled with constrained health-care budgets, has implications for surgical capacity and economic burden on health-care systems. Hence, systems need to manage patient care as efficiently as possible, maintain or improve outcomes, and reduce the economic burden of these procedures.

Enhanced recovery programs (ERPs) are clinical initiatives to optimize patient recovery following surgery, thereby reducing length of hospital stay and hospital costs. ${ }^{12}$ First described for colorectal cancer surgery in $1997,{ }^{13}$ ERPs have been increasingly adopted across multiple surgical specialties. For example, within hospitals participating in the United Kingdom's Perioperative Quality Improvement Program (PQIP), 61\% of patients undergoing elective surgery receive care through an enhanced recovery pathway. ${ }^{14}$

In the orthopedic specialty, ERPs typically include pre-, intra- and post-operative measures to improve patient engagement, enhance pathways during the cycle of care, and improve clinical outcomes in order to achieve earlier discharge, improve recovery in the home setting, and expedite return to usual activity levels. ${ }^{12,15,16}$ In the joint replacement setting, ERPs have demonstrated benefits such as reduced length of hospital stay and increased quality of life. ${ }^{17-20}$

The objective of this study was to evaluate the impact of implementing five ERP principles within a Patient Pathway Optimization (PPO) program for patients undergoing TKA or THA in Cantonal Hospital Münsterlingen, a large public hospital in Switzerland. To address this, we sought to determine whether implementation of the PPO for patients undergoing TKA or THA could (1) Reduce length of stay (LOS) during the index hospitalization, (2) Reduce hospital inpatient costs, (3) Impact the rate of complications and readmissions, and/or (4) Result in more patients being discharged to home vs alternative health-care settings.

\section{Materials and Methods Study Design}

This was a retrospective observational study comparing outcomes pre- and post-PPO program implementation for patients who underwent TKA or THA at the Cantonal Hospital Münsterlingen in Switzerland. Ethical approval for this study was obtained from the National Ethics Committee Swissethics (Project ID 2020-00897) and the study complied with the Declaration of Helsinki. No patient consent was obtained for the study due to its retrospective design. Patient consent for TKA and THA surgery was obtained as per hospital protocol.

\section{Patients}

The study population included patients who underwent primary TKA or THA at the Cantonal Hospital Münsterlingen between January 2014 and December 2019. Only patients with American Society of Anesthesiologists Physical Status Classification System (ASA) scores of 1-2 were enrolled in the PPO so as to avoid confounding of additional medical comorbidities. Patients were excluded from the analysis based on the following criteria: ASA $>2$, revision TKA/THA surgery for the index hospitalization (defined as the first hospitalization for TKA or THA), pre-planned bi-lateral primary TKA/THA for the index hospitalization, dementia, unable to read and follow written and oral instructions, TKA with higher degrees of constraints than ligament balanced joint resurfacing, TKA or THA for traumatic injuries, TKA or THA due to neoplasm, partial joint replacement procedures.

\section{PPO Program}

A PPO program (formerly known as Care4Today ${ }^{\circledR 21}$ ) was initiated by the Johnson \& Johnson Services and Solutions team at the Cantonal Hospital Münsterlingen to guide the coordination of care and standardization of key practices. The program aimed to improve the current care pathway to reduce inefficiencies in clinical practice by creating detailed process descriptions, and to increase the commitment of the multidisciplinary team by involving all relevant stakeholders in definition and implementation of the PPO. The PPO program was implemented in four phases (see Supplementary

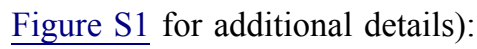


1. an initial assessment of the current (pre-PPO) pathway performance with recommendations for improvement

2. preparation for PPO implementation including analysis of the initial review and regular workshops for process optimization

3. implementation of changes creating the post-PPO pathway (on 1 April 2016)

4. follow up with assessments of key performance indicators and knowledge sharing.

Five additional enhanced recovery components were implemented:

(i) Preoperative patient education

(ii) Pre-operative physiotherapy

(iii) Local infiltration analgesia

(iv) Early mobilization

(v) Oral analgesia

Implementation of the enhanced recovery components was followed by measures for continuous improvement and audit. It should be noted that the hospital had already implemented a number of ERP principles prior to PPO introduction. Components of the ERPs implemented at the hospital pre- and post-PPO are shown in Table 1 and further details on the PPO elements are provided in Supplementary Methods and Supplementary Figure S2. The PPO program did not influence on the implants used by the hospital team; the same implants were used pre- and post-PPO implementation.

\section{Data Sources}

Electronic medical records (EMR) and billing data, which had been de-identified by the hospital staff, were retrieved from the Cantonal Hospital Münsterlingen. Medical charts and EMRs were used to extract patient variables such as demographics, outcomes, patient and procedure characteristics, as well as dates of hospital admission, discharge and re-

Table I Enhanced Recovery Principles Implemented Before and After Patient Pathway Optimization

\begin{tabular}{|c|c|c|c|}
\hline \multirow[t]{2}{*}{ Component } & \multirow[t]{2}{*}{ Pre-PPO } & \multicolumn{2}{|c|}{ Post-PPO } \\
\hline & & TKA & THA \\
\hline Preoperative information, education and counselling & No & Yes & Yes \\
\hline \multicolumn{4}{|l|}{ Preoperative optimization } \\
\hline Management of anemia & Yes & Yes & Yes \\
\hline Pre-operative physiotherapy & No & Yes & Yes \\
\hline Preoperative fasting & No & No & No \\
\hline Standard anesthetic protocol & Yes & Yes & Yes \\
\hline Use of local anesthetics for infiltration analgesia and nerve blocks & No & Yes & Yes \\
\hline Postoperative nausea and vomiting* & Yes & Yes & Yes \\
\hline Prevention of perioperative blood loss & Yes & Yes & Yes \\
\hline Perioperative oral analgesia (pre-emptive analgesia) & No & Yes & Yes \\
\hline Maintaining normothermia & Yes & Yes & Yes \\
\hline Antimicrobial prophylaxis & Yes & Yes & Yes \\
\hline Antithrombotic prophylaxis treatment & Yes & Yes & Yes \\
\hline Perioperative surgical factors & Yes & Yes & Yes \\
\hline Perioperative fluid management & Yes & Yes & Yes \\
\hline Postoperative nutritional care & No & No & No \\
\hline Early mobilization & No & Yes & Yes \\
\hline Criteria-based discharge & Yes & Yes & Yes \\
\hline Continuous improvement and audit & No & Yes & Yes \\
\hline
\end{tabular}

Notes: *For all patients where required. Bold indicates principles that were changed with the PPO program implementation. Table based on data from Wainwright et al, $2020 .{ }^{49}$ Abbreviations: PPO, patient optimization pathway; THA, Total Hip Arthroplasty; TKA, Total Knee Arthroplasty. 
admission. Billing data was used to group patients by diagnoses and procedure codes, and internal cost data informed estimates for healthcare resource utilization and costs. Inpatient costs during the index hospitalization, inpatient costs at 90 days post-admission and re-admission costs were allocated to each patient under the national standard REKOLE ${ }^{\circledR}$ full cost accounting method. ${ }^{22}$ Costs were calculated using the Swiss standard approach named REKOLE ${ }^{\circledR}$ (Revision der Kostenrechnung und Leistungserfassung - Revision of cost accounting and activity recording). In this originator-oriented cost allocation system, indirect costs (eg, operating theatre overhead) are billed to the patient from the given list of cost centers via a defined allocation formula (eg, total housing costs are divided by total number of inpatient days). It is important to note that the costs per inpatient day can therefore vary between years if total patient numbers and therefore total inpatient days differ significantly. Direct costs including medication, blood products, and medical devices and implants are directly attributed to the patient. Costs were calculated in Swiss Francs (CHF) and converted to US dollars (USD) using the exchange rate at time of writing ( $1 \mathrm{CHF}=1.09641 \mathrm{USD}$, OANDA Currency Converter) ${ }^{23}$

Complications coded according to the International Classification of Diseases (10th Revision) (ICD-10) arising directly as a result of TKA and THA procedures were identified (Supplementary Table S1) and subsequently extracted from patient records. Complications were identified when these occurred during the index hospital admission, whereas complications requiring further treatment at Cantonal Hospital Münsterlingen after the index hospitalization were considered as readmissions. Re-admission costs for patients who returned within 18 days of index admission were included within index hospitalization costs, whereas readmission costs comprised costs incurred by patients who required readmission between 19 and 90 days of index admission. Analyses for TKA and THA were conducted separately.

\section{Outcomes}

LOS during the index hospitalization (days) was the primary outcome in this study. Secondary outcomes included perpatient inpatient costs for the index hospitalization (inclusive of re-admissions within 18-days of index discharge), perpatient inpatient costs through 90 days of the index admission (which included costs of index hospitalization and any readmissions up to 90 days), complications during the index hospitalization, complications requiring readmission (up to 18 days and between 19 and 90 days post-admission), and discharge destination after index hospitalization (home or alternative health-care settings). Alternative health-care settings included rehabilitation centers, nursing homes, and hospitals other than Cantonal Hospital Münsterlingen.

\section{Sample Size and Study Power}

The study was powered to detect significant differences in the primary endpoint (LOS during the index hospitalization); all other statistical tests were considered exploratory. The calculations were based on the Wilcoxon-Mann-Whitney twosided text, alpha at 5\% and 90\% power. Sample size calculations were evaluated for TKA and THA separately with a mean baseline length of stay of 8.4 days for TKA and 8.5 days for THA (estimated by the hospital). The standard deviations were estimated to be 3.9 days for TKA and 3.9 days for THA. ${ }^{24}$ The expected reductions in length of stay were 2 days for TKA and 2.5 days for THA. ${ }^{25}$ A minimum of 67 patients per group for TKA and 42 patients for THA were necessary.

\section{Statistical Analysis}

Statistical tests were performed to compare the pre-PPO and post-PPO groups in TKA and THA cohorts separately; no statistical comparisons were made between TKA and THA groups since each was considered an independent analysis for a specific cohort of patients.

All study variables (primary and secondary endpoints) were analyzed descriptively in bivariate analyses. Counts and proportions were reported for categorical variables. Means, medians, and standard deviations were reported for all continuous variables. Statistical tests were performed to compare the differences for each outcome between the two groups (pre- and post-PPO implementation) according to the distribution of the outcomes in bivariate analyses. For nonnormally distributed continuous variables, the Mann-Whitney-Wilcoxon test was used. For continuous variables that approximately followed the normal distribution, a two-sample $t$-test was used. Chi-squared tests were used for categorical variables. Results of bivariate analyses are shown in the Supplementary Table S2. 
Multivariable generalized linear models (GLM) were constructed to examine the outcomes and adjust for covariates that may affect these outcomes. GLM with a gamma distribution and log link was used to model LOS and costs. To evaluate if the LOS was reducing naturally over time during the pre-PPO implementation period, a multivariate GLM model was built adding time as an explanatory variable in the pre-PPO group and a plot of unadjusted LOS over time was drawn. A logistic regression model was used to model discharge destination. Covariates were chosen based on a priori clinical knowledge, including patient demographics (age and sex), procedural characteristics (surgeon seniority and initial diagnosis), and clinical characteristics (body mass index [BMI] and ASA score). All analyses were conducted using R Studio version 3.6.0 (Boston, MA). Statistical significance was set a-priori at $\mathrm{p}<0.05$ (two-sided) for each analysis.

\section{Results}

\section{Patient Baseline Characteristics}

Between 2014 and 2019, 440 patients underwent TKA or THA at the Cantonal Hospital Münsterlingen and were included in the analysis.

\section{TKA Cohort}

In total, 167 TKA patients were included (122 patients pre-PPO and 45 patients post-PPO, Table 2); 35.9\% patients were male, mean age was 66.1 years (SD 9.8) and mean BMI was 30.1 (SD 5.9). The majority of patients had ASA score of 2

Table 2 Patient Demographics and Baseline Clinical Characteristics in the Total Knee Arthroplasty Cohort

\begin{tabular}{|c|c|c|c|}
\hline \multirow[b]{2}{*}{ Baseline Characteristics } & \multicolumn{3}{|c|}{ Total Knee Arthroplasty } \\
\hline & Pre-PPO & Post-PPO & Total \\
\hline $\mathbf{N}$ & 122 & 45 & 167 \\
\hline \multicolumn{4}{|l|}{ Sex, n (\%) } \\
\hline Male & $44(36.1 \%)$ & $16(35.6 \%)$ & 60 (35.9\%) \\
\hline \multicolumn{4}{|l|}{ Age, years } \\
\hline Mean, years (SD) & $66.8(9.3)$ & $64.0(10.9)$ & 66.1 (9.8) \\
\hline \multicolumn{4}{|l|}{ ASA, n (\%) } \\
\hline I & 15 (12.3\%) & $5(11.1 \%)$ & $20(12.0 \%)$ \\
\hline 2 & $107(87.7 \%)$ & 40 (88.9\%) & 147 (88.0\%) \\
\hline \multicolumn{4}{|l|}{ BMI } \\
\hline Mean (SD) & $30.6(6.2)$ & $28.5(4.7)$ & $30.1(5.9)$ \\
\hline Missing (n) & I & 1 & 2 \\
\hline \multicolumn{4}{|l|}{ Year of procedure, $\mathbf{n}(\%)$} \\
\hline 2014 & 47 (38.5\%) & - & $47(28.1 \%)$ \\
\hline 2015 & 67 (54.9\%) & - & 67 (40.1\%) \\
\hline $2016 *$ & $8(6.6 \%)$ & II (24.4\%) & 19 (11.4\%) \\
\hline 2017 & - & $12(26.7 \%)$ & $12(7.2 \%)$ \\
\hline 2018 & - & $13(28.9 \%)$ & $13(7.8 \%)$ \\
\hline 2019 & - & $9(20.0 \%)$ & $9(5.4 \%)$ \\
\hline \multicolumn{4}{|l|}{ Surgeon seniority, n (\%) } \\
\hline Chief & $16(13.1 \%)$ & $22(48.9 \%)$ & $38(22.8 \%)$ \\
\hline Senior & $106(86.9 \%)$ & $21(46.7 \%)$ & $127(76.0 \%)$ \\
\hline Junior & $0(0 \%)$ & $2(4.4 \%)$ & $2(1.2 \%)$ \\
\hline \multicolumn{4}{|l|}{ Diagnosis, n (\%) } \\
\hline Primary gonarthrosis & $107(87.7 \%)$ & $39(86.7 \%)$ & I 46 (87.4\%) \\
\hline Secondary, posttraumatic and other gonarthrosis & $13(10.7 \%)$ & $6(13.3 \%)$ & $19(11.4 \%)$ \\
\hline Other & $2(1.6 \%)$ & $0(0 \%)$ & $2(1.2 \%)$ \\
\hline
\end{tabular}

Note: *The PPO program was implemented on I April 2016.

Abbreviations: ASA, American Society of Anesthesiologists Physical Status Classification System; BMI, Body Mass Index; PPO, Patient Pathway Optimization; SD, standard deviation. 
$(88.0 \%)$ and primary gonarthrosis as their primary diagnosis $(87.4 \%)$. Patient demographics were not significantly different between the pre-PPO and post-PPO patient groups, with the exception of BMI $(p=0.041)$ and seniority of surgeon $(\mathrm{p}<0.001)$.

\section{THA Cohort}

A total of 273 THA patients were included (180 patients pre-PPO and 93 patients post-PPO, Table 3); $52.4 \%$ of patients were male, mean age was 64.7 years (SD 11.4) and mean BMI was 26.9 (SD 4.4). The majority of patients had an ASA score of $2(86.4 \%)$ and had primary coxarthrosis as their primary diagnosis (76.6\%). Patient demographics were not significantly different between the pre-PPO and post-PPO patient groups, with the exception of age $(\mathrm{p}<0.001)$ and seniority of surgeon $(\mathrm{p}<0.001)$.

\section{Study Outcomes}

Full details of multivariate analyses (covariate adjusted results) for each relevant pre-defined study outcome are presented in Table 4 for the TKA and THA cohorts; results from bivariate analyses (unadjusted) are provided in Supplementary Table S2.

\section{LOS During the Index Hospitalization \\ TKA Cohort}

In the multivariate analysis, mean LOS during the index hospitalization was 8.43 days in the pre-PPO group and 5.96

Table 3 Patient Demographics and Baseline Clinical Characteristics in the Total Hip Arthroplasty Cohort

\begin{tabular}{|c|c|c|c|}
\hline \multirow[b]{2}{*}{ Baseline Characteristics } & \multicolumn{3}{|c|}{ Total Hip Arthroplasty } \\
\hline & Pre-PPO & Post-PPO & Total \\
\hline $\mathbf{N}$ & 180 & 93 & 273 \\
\hline \multicolumn{4}{|l|}{ Sex, n (\%) } \\
\hline Male & 89 (49.4\%) & $54(58.1 \%)$ & 143 (52.4\%) \\
\hline \multicolumn{4}{|l|}{ Age, years } \\
\hline Mean, years (SD) & $66.7(11.0)$ & $60.9(11.4)$ & $64.7(11.4)$ \\
\hline \multicolumn{4}{|l|}{ ASA, n (\%) } \\
\hline 1 & $24(13.3 \%)$ & $13(14.0 \%)$ & 37 (I3.6\%) \\
\hline 2 & 156 (86.7\%) & $80(86.0 \%)$ & $236(86.4 \%)$ \\
\hline \multicolumn{4}{|l|}{ BMI } \\
\hline Mean (SD) & $26.8(4.6)$ & $27.1(3.9)$ & $26.9(4.4)$ \\
\hline \multicolumn{4}{|l|}{ Year of procedure, $\mathbf{n}(\%)$} \\
\hline 2014 & $82(45.6 \%)$ & - & $82(30.0 \%)$ \\
\hline 2015 & $90(50.0 \%)$ & - & $90(33.0 \%)$ \\
\hline $2016 *$ & $8(4.4 \%)$ & $18(19.4 \%)$ & $26(9.5 \%)$ \\
\hline 2017 & - & $29(31.2 \%)$ & $29(10.6 \%)$ \\
\hline 2018 & - & $29(31.2 \%)$ & $29(10.6 \%)$ \\
\hline 2019 & - & $17(18.3 \%)$ & 17 (6.2\%) \\
\hline \multicolumn{4}{|l|}{ Surgeon seniority, n (\%) } \\
\hline Chief & $4 \mathrm{l}(22.8 \%)$ & $42(45.2 \%)$ & $83(30.4 \%)$ \\
\hline Senior & 139 (77.2\%) & $48(51.6 \%)$ & $187(68.5 \%)$ \\
\hline Junior & $0(0 \%)$ & $3(3.2 \%)$ & $3(1.1 \%)$ \\
\hline \multicolumn{4}{|l|}{ Diagnosis, n (\%) } \\
\hline Primary coxarthrosis & $|4|(78.3 \%)$ & $68(73.1 \%)$ & $209(76.6 \%)$ \\
\hline Secondary, posttraumatic and other coxarthrosis & $25(13.9 \%)$ & $18(19.4 \%)$ & $43(15.8 \%)$ \\
\hline Necrosis & 14 (7.8\%) & 7 (7.5\%) & 21 (7.7\%) \\
\hline
\end{tabular}

Note: *The PPO program was implemented on I April 2016.

Abbreviations: ASA, American Society of Anesthesiologists Physical Status Classification System; BMI, Body Mass Index; PPO, Patient Pathway Optimization; SD, standard deviation. 
Table 4 Summary of Study Outcomes for Total Knee and Hip Arthroplasty Cohorts Pre- and Post-Patient Pathway Optimization Implementation - Multivariate Analyses

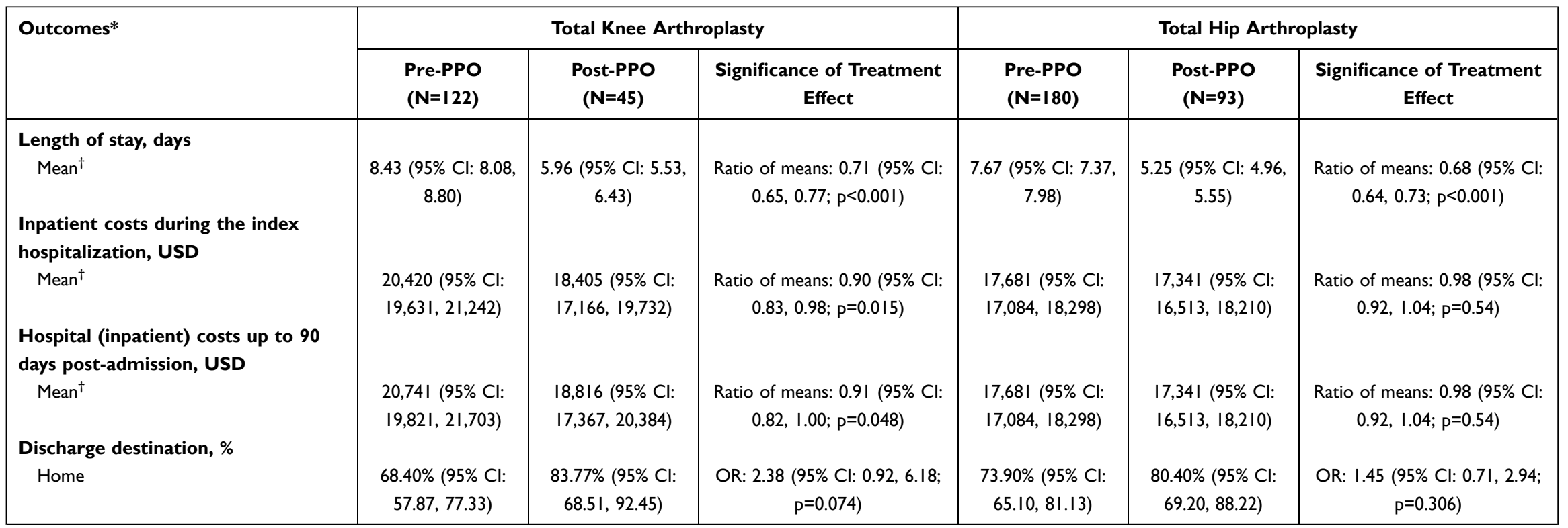

Notes: *Adjusted for age, gender, BMI, ASA score, surgeon seniority and main diagnosis (history was only available for few patients and hence not included in model); except for discharge destination, where analysis was adjusted for age, gender, BMI and ASA score only. 'Means refer to adjusted (least square) means following multivariate analysis.

Abbreviations: ASA, American Society of Anesthesiologists Physical Status Classification System; BMI, Body Mass Index; Cl, Confidence Interval; OR, odds ratio; PPO, Patient Pathway Optimization; SD, standard deviation; USD, United-States Dollar. 
days in the post-PPO group (multivariate analysis); a reduction of 2.47 days $(\mathrm{p}<0.001$; Table 4$)$. When time was added as an explanatory variable in the model for the pre-PPO patients, the slope coefficient was not different from zero $(\mathrm{p}=$ 0.2671) suggesting that the LOS was not reducing over time before PPO implementation. This could also be shown graphically (Supplementary Figure S3).

\section{THA Cohort}

Mean LOS during the index hospitalization was 7.67 days in the pre-PPO group and 5.25 days in the post-PPO group (multivariate analysis); a reduction of 2.42 days $(\mathrm{p}<0.001$; Table 4). Again, for the pre-PPO patients, the slope coefficient was not different from zero $(\mathrm{p}=0.2266)$ suggesting no significant reduction over time before PPO implementation (see also Supplementary Figure S3).

\section{Hospital Inpatient Costs \\ TKA Cohort}

Mean per-patient costs during the index hospitalization were reduced by $9.9 \%$ from USD20,420 in the pre-PPO group to USD18,405 in the post-PPO group (multivariate analysis); a reduction of USD2016 ( $\mathrm{p}=0.015$; Table 4). Mean perpatient hospital inpatient costs up to 90 days post admission were also reduced by $9.3 \%$ from USD20,741 in the pre-PPO group to USD18,816 in the post-PPO group (a reduction of USD1925 [p $=0.048]$; Table 4).

\section{THA Cohort}

Mean per-patient costs during the index hospitalization were reduced by $1.9 \%$ from USD17,681 in the pre-PPO cohort to USD17,341 in the post-PPO cohort (multivariate analysis); a reduction of USD340 [p $=0.538$ ]; Table 4. As no readmissions occurred in either the pre-PPO or post-PPO patient group between 19 and 90 days post admission, mean per-patient hospital inpatient costs up to 90 days post admission were identical to costs for the index hospitalization (Table 4).

\section{Complication Rates and Readmissions}

Multivariate analyses of complication rates were precluded by the occurrence of very few of these events; therefore, only a descriptive analysis was performed for these outcomes.

\section{TKA Cohort}

Two patients from the pre-PPO group (1.6\%) and one patient from the post-PPO group (2.2\%) had complications during the index hospitalization. No patients from either group were re-admitted due to complications within 18 days of index admission, while two patients in the pre-PPO and one patient in the post-PPO group experienced complications and were readmitted between 19 and 90 days post admission (Supplementary Table S2).

\section{THA Cohort}

Complications during the index hospitalization were reported for two patients (1.1\%) in the pre-PPO group and no patients in the post-PPO group. Two patients, one in the pre-PPO group and one from the post-PPO group, were readmitted due to complications within 18 days. Two patients (one patient in each group) were readmitted for complications between 19 and 90 days of admission (Supplementary Table S2).

\section{Discharge Location (Home vs Alternative Health-care Setting) TKA Cohort}

The proportion of patients who were discharged to home was lower in the pre-PPO group than in the post-PPO group ( $68.4 \%$ vs $83.8 \%$, respectively, multivariate analysis). The between-group (pre- vs post-PPO) odds ratio (OR) for TKA patients discharged to home vs an alternative health-care setting was $2.38(95 \%$ CI: $0.92-6.18, p=0.074$, Table 4).

\section{THA Cohort}

The proportion of patients who were discharged to home was lower in the pre-PPO group than in the post-PPO group 
( $73.9 \%$ vs $80.4 \%$, respectively, multivariate analysis). The between-group (pre- vs post-PPO) OR for THA patients discharged to home vs an alternative health-care setting was 1.45 (95\% CI: 0.71-2.94, $\mathrm{p}=0.306$, Table 4).

\section{Discussion}

Health-care budget constraints and the growing demand for joint replacements underscore a need to improve efficiency and reduce costs associated with TKA and THA procedures. In this observational retrospective cohort study, a PPO program was introduced in the care cycle of TKA and THA patients in Cantonal Hospital Münsterlingen. Additional ERP elements were selected using a multidisciplinary project team approach to reduce inefficiencies in the current care pathway as well as increase the commitment of all relevant stakeholders. The newly introduced care pathway guidelines in the hospital also reduced variation in patient treatment.

Our analysis has some limitations. The study was only powered to detect significant differences in the primary outcome and all other outcomes were therefore considered exploratory. Our study also included a selected set of patient demographics and comorbidity data; therefore, we were unable to control for all potential confounders. In particular, this study did not evaluate surgical time or blood loss, which may have impacted the clinical and economic findings. Our study was a comparison of outcomes pre- and post-implementation of the PPO, rather than a contemporaneous comparison of patients; however, although time trends were not adjusted for, there was no substantial change in LOS over time before the program implementation (Supplementary Figure S3). This research also was limited in its ability to identify all post-surgery complications, since it did not ascertain complications that patients may have presented to care providers other than the index hospital. However, TKA/THA patients who receive surgery at Cantonal Hospital Münsterlingen most commonly would present to the same hospital to address post-operative complications. Lastly, this study might be subject to bias as the sponsor also provided the PPO program.

LOS is considered a key driver of total costs of total joint arthroplasty, and is hence a commonly evaluated endpoint in ERP studies. ${ }^{26-28}$ Implementation of the PPO program for TKA and THA patients was associated with reduced LOS (decrease of 2.47 and 2.42 days in the TKA and THA cohorts, respectively). The baseline LOS following TKA and THA in the pre-PPO period may differ compared with other regions and countries (eg, the USA). However, they were similar or lower than the mean LOS across all hospitals in Switzerland during the same period. The mean LOS for TKA and THA in the pre-PPO period in our study was 8.4 and 7.7 days, respectively, while across all hospitals in Switzerland, the mean LOS was 8.9 and 8.4 days, respectively. ${ }^{29,30}$

Reduced LOS was associated with a significant inpatient cost reduction in the TKA cohort after PPO implementation (mean inpatient costs during the index hospitalization and up to 90 days post admission decreased by USD2016 and USD1925 per patient, respectively). Although the magnitude of LOS reduction after PPO implementation was similar in the TKA and THA cohorts, the difference did not translate into a significant cost reduction in the THA cohort (mean perpatient inpatient costs during index hospitalization and up to 90 days post admission both decreased by USD340). This may be due to the fact that the latter days of a patient's stay incur fewer costs compared with the initial post-operative period, as patients need less support during the latter period of their stay. In addition, the current study was powered to only detect differences in LOS and not in the secondary endpoints. The cost analysis could also have been affected by the pre/post nature of the study; captured costs could have included temporal differences as outlined above when detailing the REKOLE accounting method. However, as a line-item cost analysis was not performed, it was not possible to determine why reduced LOS translated into a cost saving for TKA patients but not THA patients. Although cost savings were not demonstrated for the THA cohort following PPO implementation, more rapid discharge of patients may have freed up more resources to treat additional patients, thereby facilitating improved use of hospital resources (however, this aspect was not assessed within our study). This study did not evaluate the extra cost and burden associated with the introduction of the PPO program which may limit the findings; further research should be conducted considering these elements. Our findings are aligned with other published reports showing that ERP implementation can lead to reduced LOS; our results also demonstrated that cost-savings may be achievable as a result of shorter LOS. ${ }^{21,31-33}$

The reduction in LOS during index hospitalization following PPO implementation was not associated with an increase in complications either during the index stay or up to 90 days post admission. 
There were more patients in the post-PPO groups who were discharged to home than to alternative health-care settings, for both the TKA and THA cohorts (OR 2.38 and 1.45, respectively). However, the differences in proportions of patients discharged to home vs alternative health-care settings were not significant in either cohort; this could be attributed to the current study being powered to only detect differences in LOS and not in the secondary endpoints. It should be noted that, in clinical practice, patient preferences are considered when determining the discharge destination. In the authors' experience, there is a tendency for patients to prefer a rehabilitation facility over home setting even if it would not be strictly required from a clinical perspective.

This study provides real-world evidence of the benefits of implementing additional enhanced recovery principles and reducing inefficiencies during the cycle of care for patients receiving TKA and THA at Cantonal Hospital Münsterlingen, a large public hospital in Switzerland. During the study period, although patient numbers for each year were small, our results showed that the decrease in LOS during the index hospitalization following PPO implementation in 2016 was sustained through to 2019 (Supplementary Figure S3). Hence, we demonstrated that implementation of selected ERP components improved patient results and that, with the appropriate deployment support, these results can be sustained over time. Indeed, post-PPO impact was monitored by including patients treated within 4 years after PPO implementation in our study. This is notably longer than the follow-up periods in other several studies evaluating the impact of enhanced

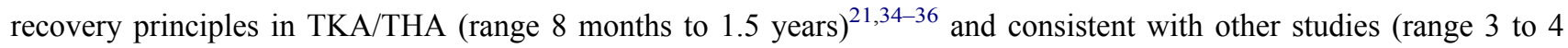
years). ${ }^{31,33}$ Cantonal Hospital Münsterlingen is a public hospital and patients were consecutively included, thereby minimizing patient selection bias, typically a limitation in observational studies. ${ }^{37}$ Our study was conducted in Switzerland, a country where rates of hip and knee replacement procedures are among the highest in Europe, and measures for efficiency gains in these procedures would be highly relevant. ${ }^{4}$

The benefits of ERPs have been demonstrated in orthopedic surgery as well as various other surgical settings; these benefits include reductions in LOS, postoperative complications, readmissions and mortality, and associated cost reductions, ${ }^{21,31-36,38-40}$ and are in line with the current findings. ERPs are adopted to accelerate patient recovery from surgery, and specific components of the protocols can influence the success of these programs. Importantly, in our study, the PPO program improved upon the existing ERP components at the hospital. We conducted a review of the published literature on ERP in orthopedics and found that such an approach has been described by other studies evaluating the impact of adding specific elements to existing ERPs, such as patient strength/resistance training, ${ }^{41,42}$ choice of analgesic${ }^{43}$ and patient education; ${ }^{33,44}$ by contrast, relatively few studies in TKA or THA patients compared the impact of an ERP with conventional care. Hence, our results demonstrate that positive impact can be achieved through the introduction of selected measured changes to patients' cycle of care. Furthermore, given the level of detail we have provided, our study should be reproducible within other centers for patients undergoing TKA and THA with ASA scores of $<3$.

The rate of ERP implementation within Europe is not currently clear; however, decreases in LOS after TKA and THA, a key aim of ERPs, have been observed over the past two decades in countries such as Spain and Denmark, ${ }^{25,45}$ suggesting increased uptake of ERP components. In the UK, enhanced recovery of patients had been deemed by PQIP as one of their national priorities for 2019-2020 with the national target for enhanced recovery pathway enrolment set at $80 \%$ of patients on elective surgery. ${ }^{14,46}$ Nonetheless, reimbursement issues and safety concerns from surgeons regarding early discharge and suitability of ERPs for certain patient groups may slow ERP implementation rates. ${ }^{47}$ Additionally, even with the adoption of ERPs, compliance levels with such programs are currently unclear. ${ }^{48}$ Therefore, in addition to promoting the ERP implementation, it is also equally important to maintain the commitment and involvement of the multidisciplinary care team such that the adopted ERP measures are achievable and sustained, as was done in our study (Supplementary Figure S3).

\section{Conclusion}

TKA and THA are clinically efficacious and cost-effective procedures; however, they represent a burden to health systems due to continued rising demand. Our study demonstrates that implementation of selected enhanced recovery principles whilst working with hospital teams to reduce inefficiency and variation in the patient pathway for TKA and THA patients may benefit both health systems and patients, by reducing LOS and costs without consequent increases in complications or readmissions. 


\section{Abbreviations}

ASA, American Society of Anesthesiologists; BMI, body mass index; EMR, electronic medical records; ERP, enhanced recovery program; GLM, generalized linear models; LOS, length of stay; OR, odds ratio; PQIP, Perioperative Quality Improvement Program; PPO, Patient Pathway Optimization; SD, standard deviation; THA, total hip arthroplasty; TKA, total knee arthroplasty.

\section{Acknowledgments}

We thank Mai Ping Tan (Mtech Access) who provided medical writing services in the preparation of the manuscript, funded by consultancy payments from Johnson \& Johnson Medical.

\section{Author Contributions}

All authors were involved in the conception (all authors), study design (all authors), execution (LE, MH, NP, NS, MS), data acquisition (NP, TG, CT) and analysis of this study (TG, CT). All authors contributed to the development or revision of the manuscript. All authors agree on the journal for submission. All authors have reviewed and agreed on the manuscript at each stage, and have approved the final version for submission. All authors have agreed to take responsibility and to be accountable for the contents of the article.

\section{Funding}

This manuscript was funded by Johnson \& Johnson Medical.

\section{Disclosure}

MH, NS, CT, TG, and HT are employees of Johnson \& Johnson Medical, who funded the manuscript. The other authors certify that he or she has no commercial associations (eg, consultancies, stock ownership, equity interest, patent/licensing arrangements, etc.) that might pose a conflict of interest in connection with the submitted article.

\section{References}

1. Organisation for Economic Cooperation and Development. Health at a Glance: Europe 2017 (Chapter 9: hip and knee replacement); 2019. Available from: https://www.oecd-ilibrary.org/docserver/2fc83b9a-en.pdf?expires=1602524184\&id=id\&accname=guest\&checksum= 8E1D95B41C5F4CBEEB4E5C5256C5013F. Accessed October 12, 2020.

2. Swiss National Joint Registry. SIRIS Report 2019. Annual Report of the Swiss National Joint Registry, Hip and Knee, 2012 - 2018 ; 2019. Available from: https://www.siris-implant.ch/de/Downloads\&category=16. Accessed September 24, 2020.

3. Carr AJ, Robertsson O, Graves S, et al. Knee replacement. Lancet. 2012;379(9823):1331-1340. doi:10.1016/S0140-6736(11)60752-6

4. Organisation for Economic Cooperation and Development. Health at a Glance: Europe 2016 (Chapter: hip and knee replacement); 2016. Available from: https://www.oecd-ilibrary.org/social-issues-migration-health/health-at-A-glance-europe-2016/hip-and-knee-replacement_health_glance_eur2016-60-en. Accessed September 24, 2020.

5. Stargardt T. Health service costs in Europe: cost and reimbursement of primary hip replacement in nine countries. Health Econ. 2008;17(S1):S9S20. doi:10.1002/hec. 1328

6. Pabinger C, Lothaller H, Portner N, Geissler A. Projections of hip arthroplasty in OECD countries up to 2050. Hip Int. 2018;28(5):498-506. doi: $10.1177 / 1120700018757940$

7. Klug A, Gramlich Y, Rudert M, et al. The projected volume of primary and revision total knee arthroplasty will place an immense burden on future health care systems over the next 30 years. Knee Surg Sports Traumatol Arthrosc. 2020;3:1-12.

8. Romanini E, Decarolis F, Luzi I, et al. Total knee arthroplasty in Italy: reflections from the last fifteen years and projections for the next thirty. Int Orthop. 2019;43(1):133-138. doi:10.1007/s00264-018-4165-7

9. Daigle ME, Weinstein AM, Katz JN, Losina E. The cost-effectiveness of total joint arthroplasty: a systematic review of published literature. Best Pract Res Clin Rheumatol. 2012;26(5):649-658. doi:10.1016/j.berh.2012.07.013

10. Pivec R, Johnson AJ, Mears SC, Mont MA. Hip arthroplasty. Lancet. 2012;380(9855):1768-1777. doi:10.1016/S0140-6736(12)60607-2

11. Price AJ, Alvand A, Troelsen A, et al. Knee replacement. Lancet. 2018;392(10158):1672-1682. doi:10.1016/S0140-6736(18)32344-4

12. Galbraith A, McGloughlin E, Cashman J. Enhanced recovery protocols in total joint arthroplasty: a review of the literature and their implementation. Irish J Med Sci. 2018;187(1):97-109. doi:10.1007/s11845-017-1641-9

13. Kehlet H. Multimodal approach to control postoperative pathophysiology and rehabilitation. Br J Anaesth. 1997;78(5):606-617. doi:10.1093/bja/ 78.5.606

14. Perioperative Quality Improvement Programme. Annual report 2017-2018; 2018. Available from: https://pqip.org.uk/FilesUploaded/PQIP\% 20Annual\%20Report\%202017-18.pdf. Accessed October 16, 2020.

15. Ghosh A, Chatterji U. An evidence-based review of enhanced recovery after surgery in total knee replacement surgery. J Perioper Pract. $2019 ; 29$ (9):281-290. doi:10.1177/1750458918791121 
16. Kaye AD, Urman RD, Cornett EM, et al. Enhanced recovery pathways in orthopedic surgery. J Anaesthesiol Clin Pharmacol. 2019;35(Suppl 1): S35. doi:10.4103/joacp.JOACP_35_18

17. Hu Z-C, He L-J, Chen D, et al. An enhanced recovery after surgery program in orthopedic surgery: a systematic review and meta-analysis. $J$ Orthop Surg Res. 2019;14(1):77. doi:10.1186/s13018-019-1116-y

18. Jones E, Wainwright T, Foster J, Smith J, Middleton R, Francis N. A systematic review of patient reported outcomes and patient experience in enhanced recovery after orthopaedic surgery. Ann R Coll Surg Engl. 2014;96(2):89-94. doi:10.1308/003588414X13824511649571

19. Judge A, Garriga-Fuentes C, Cooper C, et al. The impact of the enhanced recovery pathway and other factors on outcomes and costs following hip and knee replacement: routine data study. Health Serv Delivery Res. 2020;8(4):1-188. doi:10.3310/hsdr08040

20. Lau CS, Chamberlain RS. Enhanced recovery after surgery programs improve patient outcomes and recovery: a meta-analysis. World $J$ Surg. 2017;41(4):899-913. doi:10.1007/s00268-016-3807-4

21. Jayakumar P, Di J, Fu J, et al. A patient-focused technology-enabled program improves outcomes in primary total hip and knee replacement surgery. JBJS Open Access. 2017;2(3):e0023. doi:10.2106/JBJS.OA.16.00023

22. Besson P. REKOLE ${ }^{\circ}$ Handbuch-Betriebliches Rechnungswesen im Spital. [Rekole operational accounting in the hospital]. 4th ed. Switzerland; 2013. Bern H+ Die Spitaler der Schweiz.

23. OANDA. Currency converter; 2020. Available from: https://www1.oanda.com/currency/converter/. Accessed November $25,2020$.

24. Blonde L, Khunti K, Harris SB, Meizinger C, Skolnik NS. Interpretation and impact of real-world clinical data for the practicing clinician. Adv Ther. 2018;35(11):1763-1774. doi:10.1007/s12325-018-0805-y

25. Jimenez-Garcia R, Villanueva-Martınez M, Fernandez-de-las-penas C, et al. Trends in primary total hip arthroplasty in Spain from 2001 to 2008 : evaluating changes in demographics, comorbidity, incidence rates, length of stay, costs and mortality. BMC Musculoskelet Disord. 2011;12(1):43. doi:10.1186/1471-2474-12-43

26. Healy WL, Rana AJ, Iorio R. Hospital economics of primary total knee arthroplasty at a teaching hospital. Clin Orthopaed Related Res. 2011;469 (1):87-94. doi:10.1007/s11999-010-1486-2

27. Molloy IB, Martin BI, Moschetti WE, Jevsevar DS. Effects of the length of stay on the cost of total knee and total hip arthroplasty from 2002 to 2013. J Bone Joint Surg Am. 2017;99(5):402. doi:10.2106/JBJS.16.00019

28. Winemaker M, Petruccelli D, Kabali C, de Beer J. Not all total joint replacement patients are created equal: preoperative factors and length of stay in hospital. Can J Surg. 2015;58(3):160. doi:10.1503/cjs.008214

29. Federal Statistics Bureau. Hospital medical statistics: number of cases and average length of stay by age group, according to treatment code; 2016. Available from: https://www.bfs.admin.ch/bfs/fr/home/statistiques/catalogues-banques-donnees/tableaux.assetdetail.334599.html. Accessed January 2022.

30. Federal Statistics Bureau. Costs per case and length of stay - selection of medical and surgical type cases; 2021. Available from: https://www.bfs. admin.ch/bfs/fr/home/statistiques/catalogues-banques-donnees/tableaux.assetdetail.19524730.html. Accessed January 2022.

31. Khan SK, Malviya A, Muller SD, et al. Reduced short-term complications and mortality following enhanced recovery primary hip and knee arthroplasty: results from 6000 consecutive procedures. Acta orthopaedica. 2014;85(1):26-31. doi:10.3109/17453674.2013.874925

32. Moulton L, Evans P, Starks I, Smith T. Does a preoperative patient education programme influence outcome following elective hip and knee arthroplasty surgery? Paper presented at: Orthopaedic Proceedings; 2013.

33. Moulton LS, Evans PA, Starks I, Smith T. Pre-operative education prior to elective hip arthroplasty surgery improves postoperative outcome. Int Orthop. 2015;39(8):1483-1486. doi:10.1007/s00264-015-2754-2

34. den Hertog A, Gliesche K, Timm J, Mühlbauer B, Zebrowski S. Pathway-controlled fast-track rehabilitation after total knee arthroplasty: a randomized prospective clinical study evaluating the recovery pattern, drug consumption, and length of stay. Arch Orthop Trauma Surg. 2012;132 (8):1153-1163. doi:10.1007/s00402-012-1528-1

35. Higgins M, Jayakumar P, Kortlever JT, et al. Improving resource utilisation and outcomes after total knee arthroplasty through technology-enabled patient engagement. The Knee. 2020;27(2):469-476. doi:10.1016/j.knee.2019.10.005

36. Jones S, Alnaib M, Kokkinakis M, Wilkinson M, St Clair Gibson A, Kader D. Pre-operative patient education reduces length of stay after knee joint arthroplasty. Ann R Coll Surg Engl. 2011;93(1):71-75. doi:10.1308/003588410X12771863936765

37. Malavolta EA, Demange MK, Gobbi RG, Imamura M, Fregni F. Randomized controlled clinical trials in orthopedics: difficulties and limitations. Rev Bras Ortop. 2011;46(4):452-459. doi:10.1016/S2255-4971(15)30261-5

38. Joliat G-R, Ljungqvist O, Wasylak T, Peters O, Demartines N. Beyond surgery: clinical and economic impact of enhanced recovery after surgery programs. BMC Health Serv Res. 2018;18(1):1008. doi:10.1186/s12913-018-3824-0

39. Maempel J, Clement N, Ballantyne J, Dunstan E. Enhanced recovery programmes after total hip arthroplasty can result in reduced length of hospital stay without compromising functional outcome. Bone Joint J. 2016;98(4):475-482. doi:10.1302/0301-620X.98B4.36243

40. Paton F, Chambers D, Wilson P, et al. Effectiveness and implementation of enhanced recovery after surgery programmes: a rapid evidence synthesis. BMJ open. 2014;4(7):e005015-e005015. doi:10.1136/bmjopen-2014-005015

41. Jakobsen TL, Kehlet H, Husted H, Petersen J, Bandholm T. Early progressive strength training to enhance recovery after fast-track total knee arthroplasty: a randomized controlled trial. Arthritis Care Res. 2014;66(12):1856-1866. doi:10.1002/acr.22405

42. Mikkelsen LR, Petersen M, Søballe K, Mikkelsen S, Mechlenburg I. Does reduced movement restrictions and use of assistive devices affect rehabilitation outcome after total hip replacement? A non-randomized, controlled study. Eur J Phys Rehabil Med. 2014;50(4):383-393.

43. McDonald D, Deakin A, Ellis B, et al. The technique of delivery of peri-operative analgesia does not affect the rehabilitation or outcomes following total knee arthroplasty. Bone Joint J. 2016;98(9):1189-1196. doi:10.1302/0301-620X.98B9.36250

44. Moulton LS, Evans PA, Starks I, Smith T. Preoperative education prior to elective knee arthroplasty surgery does not change patient outcomes. Musculoskeletal Care. 2017;15(4):341-344. doi:10.1002/msc.1177

45. Husted H, Jensen CM, Solgaard S, Kehlet H. Reduced length of stay following hip and knee arthroplasty in Denmark 2000-2009: from research to implementation. Arch Orthop Trauma Surg. 2012;132(1):101-104. doi:10.1007/s00402-011-1396-0

46. Perioperative Quality Improvement Programme. Annual report 2018-2019; 2019. Available from: https://pqip.org.uk/FilesUploaded/PQIP\% 20Annual\%20Report\%202018-19.pdf. Accessed October 16, 2020.

47. Vehmeijer SB, Husted H, Kehlet H. Outpatient total hip and knee arthroplasty: facts and challenges. Acta orthopaedica. 2018;89(2):141-144. doi:10.1080/17453674.2017.1410958 
48. Simpson J, Moonesinghe S, Grocott M, et al. Enhanced recovery from surgery in the UK: an audit of the enhanced recovery partnership programme 2009-2012. BJA. 2015;115(4):560-568. doi:10.1093/bja/aev105

49. Wainwright TW, Gill M, McDonald DA, et al. Consensus statement for perioperative care in total hip replacement and total knee replacement surgery: enhanced Recovery After Surgery (ERAS ${ }^{\circledR}$ ) Society recommendations. Acta Orthopaedica. 2020;91(1):3-19. doi:10.1080/ 17453674.2019.1683790

\section{Publish your work in this journal}

ClinicoEconomics and Outcomes Research is an international, peer-reviewed open-access journal focusing on Health Technology Assessment, Pharmacoeconomics and Outcomes Research in the areas of diagnosis, medical devices, and clinical, surgical and pharmacological intervention. The economic impact of health policy and health systems organization also constitute important areas of coverage. The manuscript management system is completely online and includes a very quick and fair peer-review system, which is all easy to use. Visit http://www.dovepress.com/testimonials.php to read real quotes from published authors.

Submit your manuscript here: https://www.dovepress.com/clinicoeconomics-and-outcomes-research-journal 